\title{
Infrared images of heat fields around a linear heater in tree trunks: what can be learned about sap flow measurement?
}

\author{
Helmut TRIBUTSCH${ }^{\mathrm{a} *}$, Nadezhda NADEZHDINA ${ }^{\mathrm{b}}$, Jan CERMAK $^{\mathrm{b}}$ \\ ${ }^{a}$ Hahn-Meither Institute, Dept. Solare Energetik, 14109 Berlin, Germany \\ ${ }^{\mathrm{b}}$ Institute of Forest Ecology, Mendel University of Agriculture and Forestry, Zemedelska 3, Brno 61300, Czech Republic
}

(Received 23 September 2005; accepted 22 February 2006)

\begin{abstract}
This contribution aims at improving the understanding of sap flow measurements in trees. Infrared heat field images taken around heating needles in sap transporting tree trunks are characterized by isotherms of elliptic shape with the heating needle in the lower focus. Increasing sap flow increases the eccentricity of the elliptic heat field. This dynamics of ellipses provides a simplified experimental-mathematical approach for the understanding and evaluation of the otherwise very complicated heat transfer- and distribution-problem involved. The results obtained are used to discuss criteria for possible improved positioning patterns for needle sensors aimed for sap flow calculation using the dynamics of ellipses.
\end{abstract}

ellipse / heat dissipation method / heat field deformation method / linear heater / sensor geometry

Résumé - Images infrarouges des champs de chaleur autour d'un radiateur linéaire dans les troncs des arbres : que peut-on apprendre au sujet de la mesure du flux de sève? Cet article vise à améliorer la compréhension des mesures du flux de sève dans les arbres. Des images infrarouges prises autour des aiguilles de chauffage dans les troncs transportant la sève ont été caractérisées par des isothermes de forme elliptique avec l'aiguille chauffante dans le foyer le plus bas. L'accroissement du flux de sève accroît l'excentricité du champ de chaleur elliptique. Ces dynamique des ellipses fournissent une approche expérimentale et mathématique simplifiée pour la compréhension et l'évaluation autrement très compliquée du problème du transfert de chaleur. Les résultats obtenus sont utilisés pour juger de l'exactitude de la dissipation de la chaleur (HD) et de la déformation du champ de chaleur (HDF), des mesures techniques et discuter des critères pour une possible amélioration des modèles de positionnement des aiguilles détecteurs visant à calculer le flux de sève en utilisant les dynamiques des ellipses.

méthode de la dissipation de la chaleur / méthode de la déformation du champ de chaleur / radiateur linéaire / géométrie du détecteur

\section{INTRODUCTION}

The behavior of water in living plants is a mayor challenge for both biologists and physical chemists. A critical issue is the collection of reliable experimental data. The transport of water in trees via the cohesion tension mechanism has been discussed for more than one century, and it is supported by a lot of modern evidence $[10,20,23,24,28,30]$. But there are conflicting opinions [32] and an ongoing controversy [3]. Recently, a molecular kinetic theory has been proposed for the dynamics of cohesive (tensile) water turnover in trees [27]. It supports the cohesion-tension mechanism by showing that energy conversion via evaporative pulling of water is functioning. But it also introduces a more in-depth understanding of this remarkable mechanism: the build-up of cohesive tension is not simply a side phenomenon of ordinary water evaporation from leaves. It is not merely a process coupled to the water potential gradient, which develops between the atmospheres via the tree water conduits to the roots. The kinetic model for tensile water turnover [27] describes the water-tree system under solar irradiation as a vapor machine, which works subject to irreversible

\footnotetext{
* Corresponding author: Tributsch@hmi.de
}

thermodynamics. It functions as a self organizing system and its main properties can be mathematically derived from water interactions which consider reasonable feedback interactions via hydrogen bond dynamics between water molecules. They include self-organization of water into tensile structure, chaos (cavitation), oscillations (occasionally observed with the sap of plants) and a bi-stable state of water evaporation from the leaves. The latter was experimentally verified in [27] and demonstrates that evaporation of water from leaf structures does not follow the expectation of reversible thermodynamics, where water and vapor are in equilibrium. Evolution has designed the water conduit systems in such a way as to maintain the included water as a non-equilibrium "micro-canonical" ensemble. When water is pulled by evaporation processes and an increasing concentration of hydrogen bonds is activated (like in super-cooled water or in ice structures) autocatalysis in bond formation occurs leading to self-organization.

In the controversy on the cohesion - tension mechanism $[3,32]$ the reliability of tensile water measurement is an important issue. The new interpretation of tensile water dynamics [27] attributes to sap transport a non-linear dynamics (soft matter) behavior, which is quite different from that of ordinary water. It will equally require reliable measurements for testing 


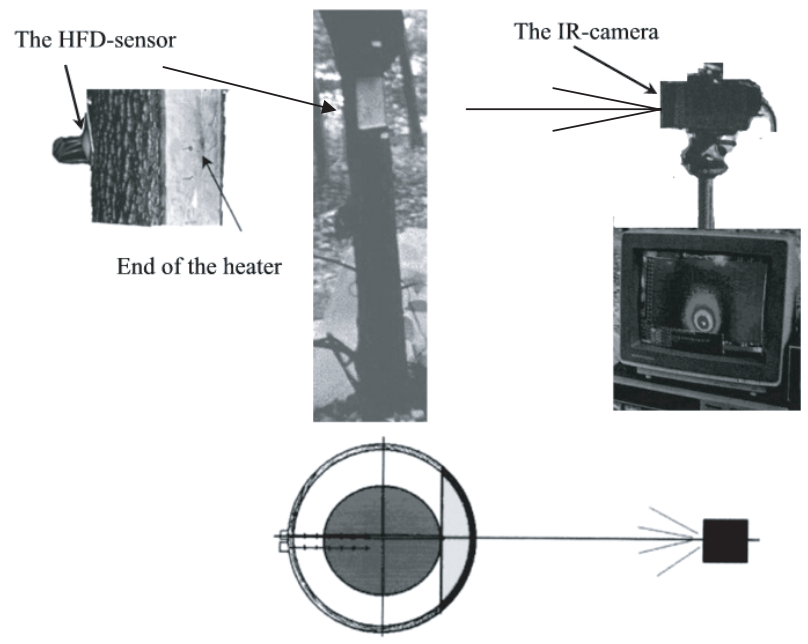

Figure 1. Experimental setup: Schemes and photo of the lime sample tree stem prepared for taking infra-red images of heat field around a linear heater visible in frontal direction: cross-section of the tree stem with the radial sap flow sensor installed from the opposite side of stem and the infra red camera focused on the smoothed stem surface. Dark area in tree trunk limits zone with similar flow rates from both opposite sides of stem (visible by infra-camera and measured between the second and third outer thermocouples of the radial sensor).

and verification. Infrared imaging techniques have been used in [27] to demonstrate via an additional experiment that water is actually pulled by evaporation. It was also shown that incident solar irradiation is intimately, but non-linearly, coupled to sap transport. Water is directly pulled by evaporative solar energy turnover.

In this publication it is attempted to show how infraredimaging techniques may help to better understand sap transport dynamics in view of optimization and improvement of traditional measurement techniques via heat sensors.

The application of thermal measurements for the analysis of water transport in trees has a long history $[1,2,4-9,11]$. They are based on a temporal or local thermal heating of sap with different strategies for the detection of the displacement of the heated liquid. Most interesting are techniques, which allow long-term xylem sap flow measurements. Besides of the heat-pulse velocity, HPV, technique, sap flow techniques with continuous recording can be divided into essentially two large groups: into methods applying the heated probe, HP, and heat balance methods $[4,6,14,21]$. The first methods are simpler but often give information on dynamics in relative units, which have to be calibrated. The latter, being more complicated, do not need calibration. The principles and comparative properties of the main sap flow methods have been reviewed $[5,22,26]$. Many types of heaters and of heat flow sensors have been studied and applied but needle heaters [7, 12, 13, 17, 31] have developed to special significance. Quite sophisticated mathematical and numerical evaluations of the heat field dynamics have been provided [9, 15, 19, 25], but evidently more information on the properties of heat fields in tree trunks is required.
In most of the mentioned HP techniques the heated and non-heated thermometers are applied (heat dissipation method, HD, [7]), in the others two main arrangements of thermocouples around the heater are used (heat field deformation method, HFD, [17]). A symmetrical one with both ends placed at equal distances up and down the heater along the axial direction, and an asymmetrical one with the upper end of the thermocouple placed at the same axial height as the heater and a lower reference, placed at a certain distance below the heater. The opinion has been expressed that symmetrical pairs of thermocouples better "feel" the low fluxes, while asymmetrical ones "feel" the middle and high fluxes.

Application of infrared (IR) cameras allowed to get direct images of the heat field comparable with sap flow rate $[1,2,8]$. Requirements to cut and smooth the stem surface seriously injure a tree and it is the main drawback of such an approach for a routine work. However, its goal is much better spatial resolution and a possibility to get the general view of the heat field when compared e.g. to the network of thermocouples installed in the sapwood, which on the other hand can be more easily recorded. Thus the IR technique is especially suitable e.g. for relatively short-term testing of methods, while limited number of thermocouples can be applied for long-term studies in almost intact trees.

In the present work, infrared thermal images of the dynamics of heat field around a heating needle will be examined with the expectation that characteristic properties can be identified, which would allow improved strategies for simple in-situ measurements.

\section{MATERIAL AND METHODS}

\subsection{Sample tree}

Lime sample tree (Tilia cordata Mill.) with diameter at breast height (DBH) equal to $15.3 \mathrm{~cm}$ was prepared for frontal image of heat field around the heater (Fig. 1). About $20 \mathrm{~cm}$ long outer part of the stem was cut off down to the depth of $26 \mathrm{~mm}$ from the southern side of stem and the opened xylem surface was smoothed by a sharp knife. The IR-camera was focused on this accordingly prepared stem surface. The radial HFD-sensor was installed from the opposite (northern) side of the stem so that the end of the long linear heater was visible on the smoothed surface and could generate the heat field for IR-images. The depth of the heated point on the smoothed surface corresponded to the xylem depth from the opposite side of the stem situated between 4 and 5 thermocouples of the radial sensor. Two small nails were fastened at the smooth surface $30 \mathrm{~mm}$ apart as reference points.

\subsection{Infra-red imaging}

Infrared images were taken by the IR-camera (Model 600 IR Imaging Radiometer from Inframetrics, 1990) with temperature resolution of $0.1{ }^{\circ} \mathrm{C}$. The temperature scale was about $4{ }^{\circ} \mathrm{C}$ within the range between 15 to $22{ }^{\circ} \mathrm{C}$. The camera was mounted on a tripod and focused on the stem of sample tree, so that the whole area of the smooth stem surface was visible on the image. 


\subsection{Sap flow measurements}

Sap flow was measured by the heat field deformation method $[16,17]$. The sensor consisted of two pairs of stainless steel needles $1.2 \mathrm{~mm}$ in diameter, each containing six pairs of differential thermocouples, and a linear (needle-like) heater. One pair of such needles was installed symmetrical at $15 \mathrm{~mm}$ distance above and below the heater, the other one at $10 \mathrm{~mm}$ distance on the side of the heater. The voltage from the thermocouples was measured and recorded every minute by the multi-channel data-logger made by UNILOG (Brno, Czechia). More detailed information about methods applied could be found in recent publication [18].

\section{RESULTS AND DISSCUSSION}

\subsection{Forms of heat field images}

Under zero sap flow conditions an elliptic pattern of isotherms was observed in the infrared image around the heating needle because heat conduction in axial direction is somewhat more favored compared to heat conduction perpendicular to it. Without sap flow and the above-mentioned wood anisotropy the ellipses should approach a circle. If they don't the ratio of the axes $a / b$ will provide information on the asymmetry of heat conduction parallel and perpendicular to the tree axis.

Because the mathematical properties of ellipses will play a mayor role in understanding heat fields, a few basic features should be sketched here:

Ellipses follow mathematical laws explained in Figure 2: They are described by two axes, $a$ and $b$, two foci, $F 1$ and $F 2$, the main limitations $A$ and $B$, the side limitations $C$ and $D$, the centre $M$. Ellipses are characterized by the fact that any point $\mathrm{P}$ on them satisfies the relation $P F 1+P F 2=2 a$, that is the distance of focus $F 1$ via point $P$ to focus $F 2$ is equivalent to the dimension of the main axis $2 a$. The distance $F 1 F 2=2 e$, that is twice the linear eccentricity $e$ of the ellipse, described by $e=\sqrt{a^{2}-b^{2}}$. The ratio of linear eccentricity to the big axis, $e / a$, is called the numerical eccentricity $\varepsilon$. The segment vertical to the main axis across the focus is called parameter $p$ with

$$
p=\frac{b^{2}}{a}
$$

Figure 3 shows how the ellipses of iso-temperature profiles change with increasing sap flow rate. While the sap flow increases the heating source is "migrating" from the centre (zero flow) to the lower focus of the ellipse (finite flow). The other focus is shifting upwards, the more the higher the sap flow rate. The reason is that the eccentricity of the ellipse is growing. At the same time the $b$-axis of the ellipse becomes smaller satisfying the condition that the eccentricity $e$ of the ellipses is increasing while, to a first approximation, the area included by an isotherm remains constant. Intuitively one can imagine that the heat cannot progress so far away perpendicular to the sap flow axis, because it is transported along with the sap.

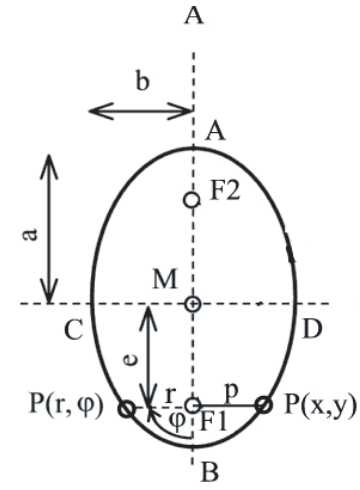

Figure 2. (A) Mathematical representation of an ellipse in standard (right part of an ellipse) and polar (left part of an ellipse) system of coordinates: $M, F 1, F 2$ - center and foci of an ellipse; $a, b$ and $e-$ main axes and eccentricity of an ellipse; $r$ and $\varphi$ polar coordinates of an ellipse. (B) Mathematical law of an ellipse: the distance between the two foci via any point on the ellipse is constant.

When the sap is transported along the $x$ - axis the original ellipse equation

$$
\frac{x^{2}}{a^{2}}+\frac{y^{2}}{b^{2}}=1
$$

transforms into

$$
\frac{\left(x-a_{0}\right)^{2}}{a^{2}}+\frac{y^{2}}{b^{2}}=1,
$$

with a simultaneous change of its numerical eccentricity $\varepsilon$, which is

$$
\varepsilon=\frac{\sqrt{a^{2}-b^{2}}}{a}=\sqrt{1-\left(\frac{b}{a}\right)^{2}} .
$$

This means: the ratio $b / a$ (the ratio of small to big axis) is changed due to the sap flow. As a consequence the centre of the ellipse $M$ shifts along the $X$ axis (by $a_{0}$ ), after the focus $F 1$ has become identical with the heat source.

Figure 3 (drawing to the right) shows how a change of sap flow rate and thus of eccentricity will influence the isotemperature profiles.

Intuitively the heat conduction process from the heat source can be understood in the following way. Heat is spreading like a wave in all directions and from every heated point heat may spread again radially. Every point on the ellipse satisfies the conditions that the distance between the two foci via this point is constant. The heat needs the same time period to travel from focus to focus of the ellipse via points on the ellipse itself (Fig. 2B). If now the sap flow changes, the heat will be displaced and the second focus shifts accordingly. This explains the shift, with increasing sap flow, of the iso-temperature ellipses in Figure 3.

There are, of course, some complications, which will have to be considered for obtaining more reliable information via the ellipse dynamics. Ideally, the heat contained within the sap filled area (which is $a b \pi$, the product of axes $a$ and $b$, multiplied by $\pi$ bordered by an isotherm should be constant. However, an elongated ellipse reflects through-flowing sap. This sap has constantly to be heated up which may result in 
a somewhat contracted isotherm ellipse depending on the rate of sap flow. There are additional complications: When carefully looking at the thermograms (Fig. 3B) one realizes that the ellipses, even though their shapes are very regular, show one peculiarity. The distance between the isothermal profiles becomes bigger around the focus which is more distant from the heating source. These ellipses are apparently distorted along the axis of sap flow. The reason may be understood: The tree tissue has a heat storage capacity and increases its temperature. It maintains better the temperature around the distant focus than around the close focus where cool sap is transporting the heat away. From the distortion between the iso-temperature profiles it may be possible to deduce heat storage parameters. Also heat diffusion or convection could act into the same direction. However, the tensile state of water, which is stronger linking water molecules via hydrogen bonds, and can transmit mechanical force, may limit such mechanisms. Nevertheless, experimentally this distortion of the ellipse form leads to limitations for sensor arrangement: they should apparently better be placed closer to the heater. Future improved theoretical models may attempt to consider such distortions.

\subsection{Theoretical analysis}

In order to understand how to evaluate infrared heat field patterns for a better planning and handling of sap flow measurements based on a minimum of sensors, some physical and mathematical considerations are required. The entire problem of combined heat and mass transport in an inhomogeneous environment such as a tree trunk is far too complicated for a rigorous evaluation (which the authors have attempted using an advanced hydromechanical computer program). Therefore we will concentrate on understanding the dynamics of the observed infrared ellipse patterns, focused around the inserted heater, the eccentricity of which changes with the magnitude of sap flow. This is seen in Figure 4, where three examples of the heat field images are shown as snapshots for the sap flow dynamics during one particular day. It was August 11th, 1999, when a more than $90 \%$ solar eclipse was shadowing the lime tree at $1 \mathrm{pm}$. This eclipse is seen as a clear dip in the continuous sap flow recording in Figure 4, which clearly shows the effect of solar radiation on evaporation, where the ambient temperature did not change by more than $2{ }^{\circ} \mathrm{C}$. The heat field ellipses measured before and afterwards (time positions 1 and 2) are comparable, due to the comparable sap flow rate. However the tree temperature was slightly higher in the afternoon, which led to a shift in the temperature color code. In the late evening the heat field (time position 3 ) has contracted from an elongated ellipse to a contracted one approaching a circle. What can be learned from the analysis of such ellipses?

\subsubsection{Evaluation of sap flow from infrared heat field images}

There are basically two phenomena involved in the dynamics of the ellipses: The first is a thermal flux via thermal con- duction $J_{T}$, which is described by the equation $(\lambda$ is the heat transfer coefficient):

$$
J_{T}=-\lambda \frac{\mathrm{d} T}{\mathrm{~d} x}=-\lambda \operatorname{grad} T
$$

The second is a thermal flux via sap transport, $J_{T S}$, which is determined by the gradient of water potential $\Psi$

$$
\left(\mathrm{d} c_{w}\right) / \mathrm{d} x=\operatorname{grad} \Psi
$$

( $c_{w}=$ water concentration, $\Psi=$ water potential, $x=$ distance $)$

multiplied with an effective diffusion constant $D$, which considers the effective friction in the Xylem water conduits, and the heat $\mathrm{H}$ (in Ws $\mathrm{mol}^{-1}$ ) which is turned over at the heater needle:

$$
J_{T S}=-H D \operatorname{grad} \Psi=-H J_{S} .
$$

Here $J_{S}$ is the sap flux. When the heat transport contributions determine the dynamics and shape of the heat field, they have to be related to dimensions within the elliptic heat field. Let us concentrate on the distance between the central heating needle and a selected isotherm of temperature $T_{S}$ parallel to the sap flow direction. In absence of sap flow this axial distance should be named $a_{a x}$. In presence of sap flow there will be thermal conduction along a similar distance $a_{a x}$, but in addition there will be a displacement of heat corresponding to the distance between the two foci of the ellipse. It corresponds to the two focal lengths $2 e=2 \sqrt{a^{2}-b^{2}}$, where $a$ and $b$ are the small and large axes of the ellipse respectively. It is the distance, which makes the difference between absence of flux and presence of flux:

$$
\frac{J_{T S}}{J_{T}}=\frac{J_{S} H}{J_{T}}=\frac{D H \operatorname{grad} \Psi}{\lambda \operatorname{grad} T}=\frac{\left(2 e+a_{a x}\right)}{a_{a x}}
$$

from this relation one can deduce:

$$
J_{T S}=-H J_{S}=-D H \operatorname{grad} \Psi=-\frac{\left(2 e+a_{a x}\right)}{a_{a x}} \lambda \operatorname{grad} T
$$

When the heat transfer coefficient $\lambda$ is provided with a dimension of $\left(\mathrm{Wm}^{-1} \mathrm{~K}^{-1}\right)$, the heat $H$, which is transferred to the sap with a dimension of $\left(\mathrm{Ws} \mathrm{mol}^{-1}\right), \operatorname{grad} T$ with a dimension of $\left(\mathrm{km}^{-1}\right)$, then the sap flow $J_{s}$ will be equivalent to and have a dimension of

$$
J_{S}=-\frac{\left(2 e+a_{a x}\right)}{a_{a x}} \frac{\lambda \operatorname{grad} T}{H}\left(\frac{m o l}{m^{2} s}\right) .
$$

From relation (7) it should be remembered, that the heat $H$ with the dimension $\mathrm{Ws} \mathrm{mol}^{-1}$ has been defined as the negative ratio of heat transport via sap transport $J_{T S}$ to the sap flux $J_{S}$. It can be assumed that the heat flux at the heat probe is increasing proportional to the provided electrical heating power $P_{H}$ and to the concentration of passing water, the sap flux $J_{S}$. The heat can therefore be written as

$$
H=\frac{k P_{H} J_{S}}{J_{S}}=k P_{H}
$$


A

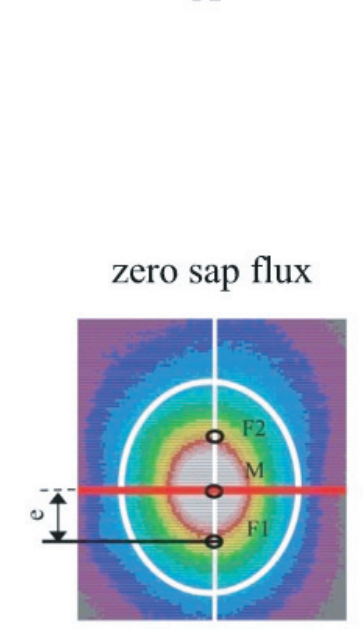

$\mathrm{B}$

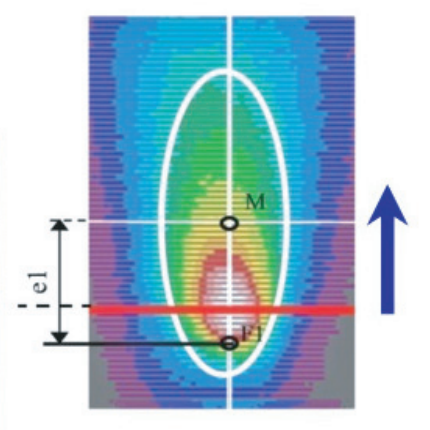

$\mathrm{C}$

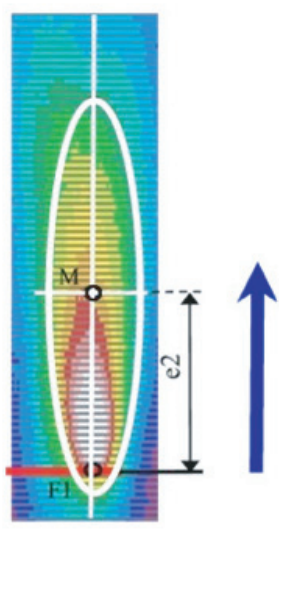

Figure 3. (A) IR-image of the heat field in the stem xylem around a linear heater under zero-flow conditions: $M-$ center of an ellipse, $F 1, F 2$ - foci of an ellipse and $e$-its eccentricity. Red horizontal line passes through axes of the heater and center of an ellipses (iso-temperature profiles). (B) and (C) IR-images of the heat field with increasing sap flow rates (shown by blue arrows). Red horizontal line marks the axis of the heater which position gradually moves with increasing flow rates from the center M (at zero-flow conditions) towards the first focus F1. Area, limited by the same isotherm, remains constant, while eccentricity of ellipses increases with increasing sap flow rates.
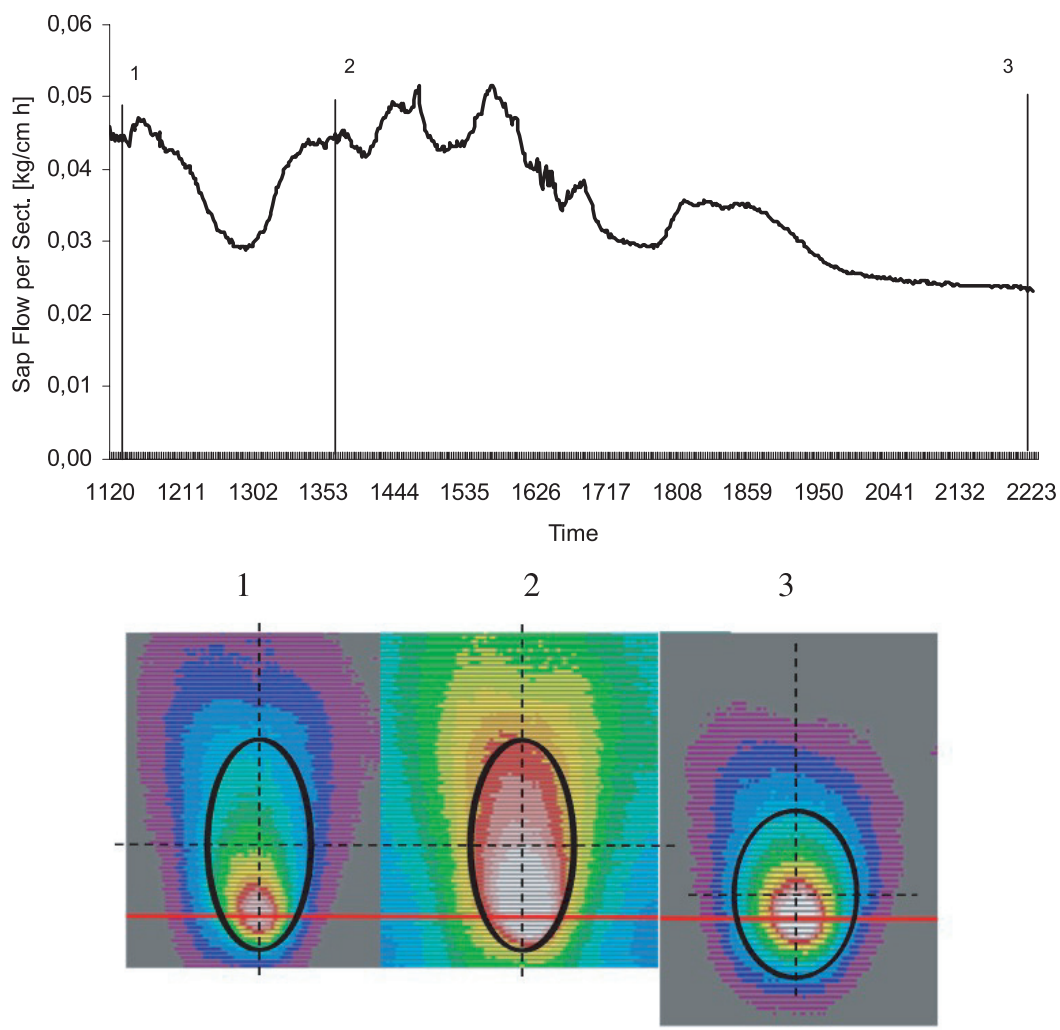

Figure 4. First two infrared images of heat field were recorded under the same flow rates at $11 \mathrm{~h} 30 \mathrm{~min}$ (left) and at $14 \mathrm{~h} 00 \mathrm{~min}$ (middle). Shape of iso-temperature profile (thick curve line) with temperature equal to $17.7^{\circ} \mathrm{C}$ (left image) is identical to that with temperature equal to $20^{\circ} \mathrm{C}$ (right image) and was characterized by the same eccentricity of ellipse. Increase of temperature was caused by increase of stem temperature. Comparably visible range of iso-temperature profiles (compare upper parts of ellipses) demonstrates similar temperature differences, corresponding to similar sap flow rates. Infrared image of the heat field (right image) was made at $22 \mathrm{~h} 22$ min (vertical line 3 ) under the same stem temperature as in the morning (vertical line 1). No one iso-temperature profiles in the right image correspond to those demonstrated on the left and middle images. Iso-thermal profile, limited by the ellipse with the area equal to those on the left and middle images, is characterized by lower eccentricity. 


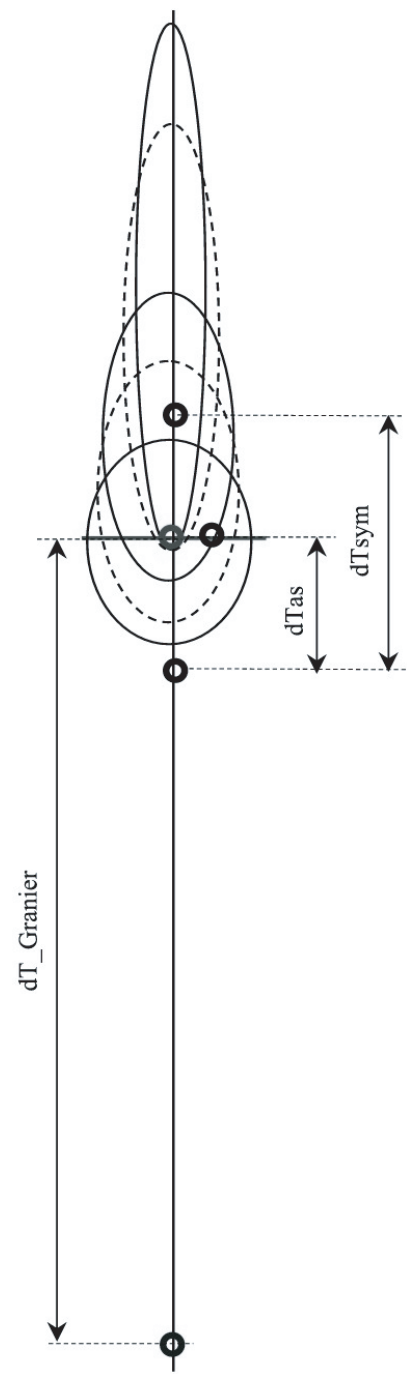

Figure 5. Set of ellipses with increasing eccentricities corresponding to increasing sap flow rates. Isotherms with equal area are presented. Positioning differential thermocouples according different methods is shown as follows: HD-method measures dT_Granier; HFD-method measures dTsym and dTas.

( $k$ is a proportionality factor determined by geometry and hydrodynamic conditions) and the sap flux becomes:

$$
J_{S}=\frac{\left(2 e+a_{a x}\right)}{a_{a x}} \frac{\lambda \operatorname{grad} T}{k P_{H}}\left[\frac{m o l}{m^{2} s}\right] .
$$

This relation now has to be interpreted. It contains dimensional parameters of the ellipses developing under negligible and given sap flow. They can be provided in real dimensions (for a given isotherm), since they cancel out. The formula also contains the heat transfer coefficient $\lambda$, which has to be provided parallel to the tree axis. The heating power $P_{H}$ transferred should be the real power loss or power turnover of the heating needle in Ws per mol of sap. In order to simplify the measurement this value $P_{H}$ should be kept constant during the experiment. It remains to be examined how the temperature gradient has to be determined. Since it controls heat conduction one should take the temperature difference between the heating needle and the selected temperature isotherm divided by the axial distance $a_{a x}$.

Equation (12) makes basically sense because the sap flow rate is, as Figure 5 shows, indeed essentially reflected in the elongation of isothermal ellipses from their thermal focus. The more power $P_{H}$ is being introduced through the heating needle, the bigger will also become the temperature gradient $\operatorname{grad} T$.

A comparatively simple way of handling Equation (12) during measurements involves the stable control of the quantity $P_{H}$, the power turnover in the heat probe, with the dimension of Ws mol${ }^{-1}$. It should remain constant, regardless of the amount of sap transported, which takes away the heat. This could be reached by Equation (1) controlling the power input electronically or (2) by applying sensor materials with electrical resistances which do not change with temperature. Examples are Konstantan $(55 \% \mathrm{Cu}, 45 \% \mathrm{Ni})$ or Manganin $(86 \% \mathrm{Cu}$, $12 \% \mathrm{Mn}, 2 \% \mathrm{Ni}$ ). Then under constant voltage input the power turnover will remain also constant. The latter is usually applied in present heat probe sensors. A constant thermal energy input has an advantage with respect to variations of sap flow during the day in different tree rings in certain tree species. This kind of sap flow variations does not affect the energy turnover and thus the applicability of Equation (12). Heat sensors placed in different depth should give consistent information on the sap transport profile.

\subsubsection{Analysis of sensor techniques from point of view of ellipse theory}

Figure 5 compares the placement of heat sensors in the HD [7] and the HFD [17] techniques. It is seen that for catching the dynamics of ellipses, the sensors are not ideally placed. The sensors placed below the heater do not appear to catch much of the changes. The asymmetric sensor placed horizontally from the heater will be exposed to a strong change of temperature, but at a very high sap flux rates it may be left outside the main thermal dynamics.

The sensors above the heater may on the other hand be left in a quite indifferent region between the foci of the isothermal ellipses.

What could, in fact, be a reliable strategy towards a reasonably accurate continuous determination of sap flow rates through the dynamics of ellipses?

Since it could be shown that the dynamics of ellipses of isotherms can give access to sap flow monitoring, ways should be found to determine the parameters of these ellipses. For long-term sap flow monitoring the challenge obviously is therefore to make temperature measurements, in an as simple as possible arrangement, which allow to determine quantitatively the axes $a$, and $b$ of the ellipse and thus the eccentricity $e$ (compare Fig. 2). In this way the complete ellipses could be determined.

The simplest approach may possibly be the following: one sensor horizontally displaced from the heating needle is needed as well as a temperature sensor array along the axis 


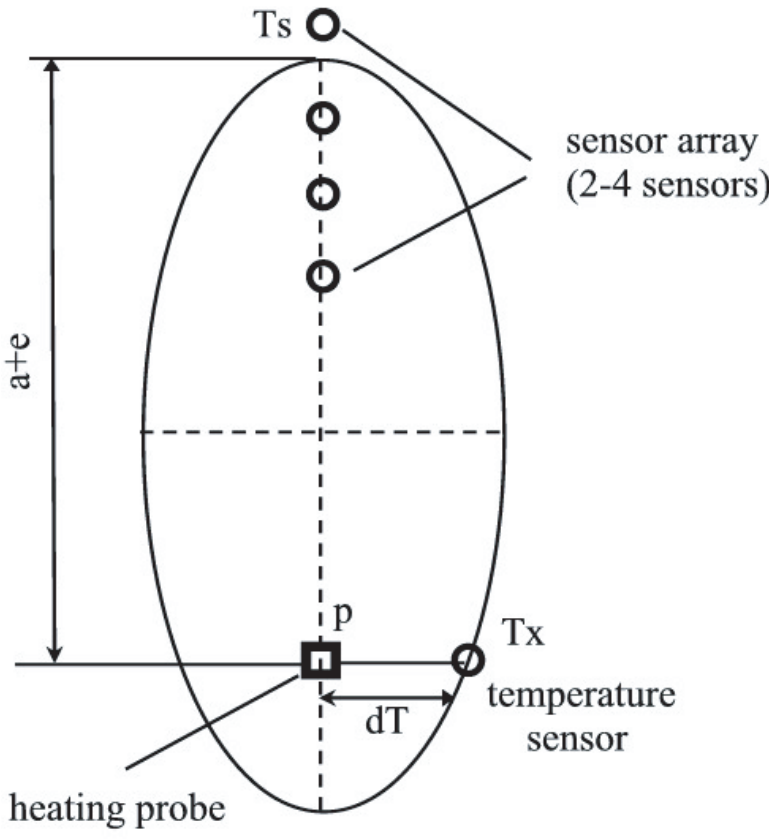

Figure 6. Simplified scheme explaining the proposed positioning of temperature sensors for determination of the parameters needed for calculating ellipse shaped isotherm.

vertically above the heating needle. This could be a series of heat sensors (at least two of them), which are wired in such a way that they can measure temperature as a function of distance (Fig. 6). The temperature would be determined along this array and a simple linear interpolation could be made to determine at what position above the heater the temperature corresponds to that measured horizontally from the heater (distance $p$ given in Eq. (1)).

The measurement problem would be relatively simple: the heat sensor at the level of and horizontally displaced from the heating needle measures a temperature $T_{x}$ and along the array in direction of the axis the position of the same temperature $T_{x}$ has to be located.

As seen from Figure 6 we have thus recognized the form of the ellipse: the horizontal distance of the temperature sensor from the heating needle gives the segment $p=b^{2} / a$ and the distance of the $T_{x}$ position above the needle gives the distance

$$
a \text { (half main axis })+e(\text { eccentricity })=z=a+\sqrt{a^{2}-b^{2}} .
$$

We have thus two measured distances and two variables, $a$ and $b$. This means, the ellipse is thus fully determined as well as all other ellipses describing isothermal lines with different temperatures.

From (1) it follows:

$$
b^{2}=p a .
$$

Inserted into (11) it follows

$$
z=a\left(1+\sqrt{1-\frac{p}{a}}\right) .
$$

Since $p$ and $z$ are measured distances, $a$ can be calculated numerically from (15) and inserted into (14) for calculation of $b$.

Basically, the HFD technique may be easier changed for measuring dynamics of ellipses in such a way that the sensor below the heater is displaced to a position above the heater and one to three more sensors are added along the axis of the tree trunk.

With a small computer program, which determines the temperature at the asymmetrical sensor, horizontally displaced from the heater, and thereafter determines the position of the same temperature at the sensor array, the necessary calculations to determine the temperature profiles for the measured temperature and of all other temperatures around the heating needle could be performed in a straightforward way. In this way the needed quantity $e$, the eccentricity, could be calculated and monitored so that the sap flow determined by relation (12) can be found (after $a_{a x}$, the horizontal distance between the central heater and the horizontally displaced sensor is inserted and $\left.\operatorname{grad} T=\left(T_{P}-T_{x}\right) / a_{x}\right)$, the temperature gradient, is determined at zero sap flow conditions).

There are also other sensor geometries imaginable, which may allow determination of the dynamic shapes of ellipses, which reflect the sap flow patterns. They appear to be more complicated. It may also be possible to design a measurement system in which the power loss at the central heater $\mathrm{k} P_{H}$ (Eq. (12)) is not kept constant but electronically measured, so that the sap flow $J_{S}$ can be computed. Only experience with the newly to be developed hardware will show what degree of perfection these proposed improved sap measurement techniques may develop.

In conclusion it may be summarized that more accurate and more reliable experimental methods are needed to monitor cohesion-tension water dynamics for overcoming controversial discussions. The dynamics of heat transport in the morphologically complex environment of the sap-transporting tree Xylem is highly complex. Experiments combining heat sensors and heat field imaging have opened a reasonable path towards handling the problem, as shown in this publication, and added to the notion that sap water in trees is actually pulled [27]. As the presented results and discussions have shown, the empirical positioning of heat sensors in conventional HD [7] and HFD [17] measurement approaches is not optimal in context of the theory of ellipses and with respect to a rational understanding of the theoretical background of measurements. The presented concepts provide for the first time a mathematical-physical basis for understanding the measurements. However, new measurement hardware has to be developed and tested for a comparative quantitative evaluation. This will be attempted in a forthcoming paper.

Acknowledgements: This study was performed within a project of the Hahn-Meitner Institute and partially within the framework of WATERUSE project (EVK1-CT-2000-00079). 


\section{REFERENCES}

[1] Anfodillo T., Sabatti M., Sigalotti G.B., Valentini R., An application of infrared thermal image to monitor water transport in plants, in: Carlomagno G.M., Corso C. (Eds.), Advanced Infrared Technology and Applications, Firenze, 1992, pp. 427-437.

[2] Anfodillo T., Sigalotti G.B., Tomasi M., Semenzato P., Valentini R., Application of thermal imaging in the study of sap flow in woody species, Plant Cell Environ. 16 (1993) 997-1001.

[3] Angeles G. et al., The Cohesion-Tension Theory, New Phytologist, Forum, www.newphytologist.org.

[4] Čermák J., Deml M., Penka M., A new method of sap flow rate determination in trees, Biol. Plant. 18 (1973) 105-110.

[5] Cohen Y., Thermoelectric methods for measurement of sap flow in plants, in: Standhill G. (Ed.), Advances in Bioclimatology, Springer-Verlag, 1993, pp. 63-89.

[6] Daum C.R., A method for determining water transport in trees, Ecology 48 (1967) 425-431.

[7] Granier A., A new method to measure the raw sap flux in the trunk of trees, Ann. For. Sci. 42 (1985) 193-200.

[8] Granier A., Anfodillo T., Sabatti M., Cochard H., Dreyer E., Tomasi M., Valentini R., Breda N., Axial and radial water flow in the trunks of oak trees: a quantitative and qualitative analysis, Tree Physiol. 14 (1994) 1383-1396.

[9] Groot A., King K.M., Measurement of sap flow by the heat balance method: numerical analysis and application to coniferous seedlings, Agric. For. Meteorol. 59 (1992) 289-308.

[10] Holbrook N.M., Burns M.J., Field C.B., Negative xylem pressures in plants: a test of the balancing pressure technique, Sci. 270 (1995) 1193-1194.

[11] Huber B., Schmidt E., Weitere thermoelektrische Untersuchungen uber den Transpirationsstrom der Baume, Tharandter Forstliche Jahrsblad 87 (1936) 369-412.

[12] Ittner E., Der Tagesgang der Geschwindigkeit des Transpirations stromes im Stamme einer 75-jahrigen Fichte. Oecol. Plant. 3 (1968) $177-183$.

[13] Karmanov V.G., Ryabova E.P., Instrument for registration of relative sap flow rate in plant, Ann. Rev. Agronom. Physics, Leningrad, 16 (1968) 81-87 (in Russian).

[14] Kucera J., Čermák J., Penka M., Improved thermal method of continual recording the transpiration flow rate dynamics, Biol. Plant. 19 (1977) 413-420.

[15] Marshall D.C., Measurements of sap flow in conifers by heat transport, Plant Physiol. 33 (1958) 385-396.

[16] Nadezhdina N., Cermák J., The technique and instrumentation for estimation the sap flow rate in plants, Patent No. 286438 (PV-158798), 1998 (in Czech).
[17] Nadezhdina N., Cermák J., Nadezhdin V., Heat field deformation method for sap flow measurements, in: Cermák J., Nadezhdina N. (Eds.), Measuring Sap Flow in Intact Plants, Proc. of 4th Int. Workshop, Zidlochovice, Czech Republic, IUFRO Publications, Brno, Czech Republic, Publishing House of Mendel University, 1998, pp. 72-92.

[18] Nadezhdina N., Tributsch H., Cermák J., Infra-red images of heat field around a linear heater and sap flow in stems of lime trees under natural and experimental conditions, Ann. For. Sci. 61 (2004) 203-213.

[19] Pickard W.F., Puccia C.J., A theory of the steadystate heat step method of measuring water flux in woody plant stems, Math. Biosci. 14 (1972) $1-15$

[20] Pockman W.T., Sperry J.S., Oleary J.W., Sustained and significant negative water-pressure in xylem, Nature 378 (1995) 715-716.

[21] Sakuratani T., A heat balance method for measuring water flux in the stem of intact plants, J. Agric. Meteorol. (Japan) 37 (1981) 9-17.

[22] Smith D.M., Allen S.J., Measurement of sap flow in plant stems, J. Exp. Bot. 47 (1996) 1833-1844.

[23] Steudle E., Trees under tension, Nature 378 (1995) 663-664.

[24] Steudle E., The cohesion-tension mechanism and the acquisition of water by plant roots, Ann. Rev. Plant Physiol. Mol. Biol. 52 (2001) $847-875$.

[25] Swanson R.H., Numerical and experimental analysis of implantedprobe heat-pulse theory, Ph.D. thesis, University Alberta, Canada, 1983.

[26] Swanson R.H., Significant historical developments in thermal methods for measuring sap flow in trees, Agric. For. Meteorol. 72 (1994) 113-132.

[27] Tributsch H., Cermak J., Nadehdina N., Kinetic studies on the tensile state of water in trees, J. Phys. Chem. 109 (2005) 17693-17707.

[28] Tyree M.T., The Cohesion-Tension theory of sap ascent: current controversies, J. Exp. Bot. 48 (1997) 1753-1765.

[29] Tyree M.T., The ascent of water, Nature 423 (2003) 923-923.

[30] Tyree M.T., Cochard H., Vessel content of leaves after excision: a test of the Scholander assumption, J. Exp. Bot. 54 (2003) $2133-2139$

[31] Vieweg G.H., Ziegler H., Thermoelektrische Registrierung der Geschwindigkeit des Transpirationsstromes, Ber. Deut. Bot. Ges. 73 (1960) 221-226.

[32] Zimmermann U., Schneider H., Wegner L.H., Haase A., Water ascent in tall trees: does evolution of land plants rely on a highly metastable state? New Phytol. 162 (2004) 575-615. 